\title{
The evolution of actinomycetes: papers from the 16th International Symposium on the Biology of Actinomycetes
}

\author{
Paul A. Hoskisson • Amanda L. Jones • \\ Gilles P. van Wezel · Francisco Barona-Gómez
}

Published online: 11 September 2012

(C) Springer Science+Business Media B.V. 2012

As with any biological system, the actinomycetes, as well as the vibrant community behind their chemical and biological study, constantly evolve. Evidence of this evolutionary process was witnessed last December 11th-15th, in Puerto Vallarta, Mexico, where the 16th International Symposium on the Biology of Actinomycetes (ISBA16) took place. After more than three decades, ISBA meetings came back to Latin America to be hosted for the first time by Mexican actinobacteriologists. The hospitality, cultural heritage and natural beauties of the geography and people from Mexico were indulged by more than 300 delegates, coming from 30 different countries, for

P. A. Hoskisson

Strathclyde Institute of Pharmacy and Biomedical

Sciences, University of Strathclyde, Glasgow, UK

A. L. Jones

Faculty of Health and Life Sciences, Northumbria

University, Newcastle Upon Tyne, UK

G. P. van Wezel ( $\square)$

Molecular Biotechnology, Institute of Biology,

Leiden University, Einsteinweg 55,

Leiden 2333CC, The Netherlands

e-mail: g.wezel@chem.leidenuniv.nl

F. Barona-Gómez ( $₫)$

Evolution of Metabolic Diversity Laboratory, Laboratorio

Nacional de Genómica para la Biodiversidad (Langebio),

Cinvestav-IPN. Km 9.6 Libramiento Norte,

CP36821 Irapuato, Mexico

e-mail: fbarona@langebio.cinvestav.mx almost a week. Indeed, surrounded by the mountains and the Pacific Ocean, together with great facilities and fantastic food, Vallarta turned out to be the perfect setting for a thrilling, but yet relaxing, winter ISBA meeting. The meeting follows on the inspiring ISBA15 meeting organized by Zixin Deng in Shanghai in 2009.

In response to environmental changes of the scientific niche colonized by the ISBA community, a major trait of ISBA16 was the fact that a myriad of recently established young scientists, with Francisco (Paco) Barona-Gómez serving as chair, was in charge of the organization of the meeting. This team of young scientists, together with well-renowned scientists from all over the world, put together an exciting scientific program that included both deep-rooted as well as recently diverging branches of actinomycete research. Sadly, however, but which also speaks volumes regarding the healthy evolutionary process, some of the scientific founders of the field, including, Professors Michael Goodfellow, David Hopwood, Julian Davies, Keith Chater and Alan Ward, were missed, in some cases, for the first time in decades.

Indeed, the ISBA16 scientific program provided both a scientific forum where the fundamental grounds of actinomycete biology, within a historical perspective, could be presented; but also new approaches and views that will certainly lead to radiation of the community were covered. This combination of perspectives provided a perfect introduction into actinomycete research for the copious number of young 
scientists that attended ISBA16. Indeed, the program included, for the first time, a plenary young scientists session (graduate students and postdoctoral fellows) consisting of 12 talks that were selected from the abstracts by a committee unrelated to the organizing committee. This session preceded the closing lecture by a veteran and leading figure of ISBA community, Professor Mervyn Bibb (John Innes Centre), who provided genomic insights into novel biosynthetic, regulatory and immunity mechanisms of lantibiotics in actinomycetes.

The ISBA16 scientific program was divided into seven major simultaneous symposia covering traditional aspects of actinomycetes research, namely: Chemistry and Biology of Natural Products; Regulatory Networks; Environmental Microbiology; Developmental Program; Pathogenic Actinobacteria; Genomics; and Systematics and Taxonomy. Each one of these symposia included a plenary lecture, which together with two ISBA16 opening plenary lectures from leading figures working with actinobacteria, but that had never attended ISBA meetings, provided a high-calibre scientific program. Professors Chris Walsh (Harvard) and Gerry Wright (McMaster) set a perfect start to the meeting by presenting breakthroughs on heterocyclic natural products biosynthesis and evolution of antibiotic resistance in the environment, respectively.

In addition, a series of specialized symposia were introduced for the first time at an ISBA meeting. These included smaller sessions on Corynebacterium; Antibiotic Resistance; Actino-tools; Plant-actinobacteria Interactions; Emerging Actinobacterial Pathogens; and 'Actinoindustriae', where speakers from industry presented specific examples on the biotechnology of actinomycetes. These new sessions will hopefully facilitate colonization of novel niches that will become part of future ISBA meetings. Many speakers from these communities attended an ISBA meeting for the first time.

Springer on behalf of Antonie van Leeuwenhoek once again generously supplied the prizes for the best posters. ISBA16 saw two prizes awarded under the names of two influential stalwarts of the field; The Professor Sir David Hopwood Prize for posters presented in the field of 'Genetics and Secondary Metabolism' and the Professor Michael Goodfellow Prize for posters in the field of 'Microbial Systematics and Ecology'. The winner of the Hopwood Prize was Hiroshi Otani from the University of Tokyo for his poster on a novel sigma factor. The winner of the Goodfellow Prize was Natalie Millan from the Scripps Institute for her poster on Salinispora ecology and evolution.

This special issue provides a selection of papers that cover the whole range of topics presented at ISBA16. We hope you enjoy this collection of diverse and in-depth papers as much as we did by taking part in the editorial process. We would like to thank authors, reviewers and editors for their hard work to make this issue happen, as well as the Antonie van Leeuwenhoek journal for providing a home for an ISBA special issue once again. Hopefully, this issue will open your appetite to attend ISBA17. At ISBA16, Professor Gilles van Wezel was elected as the new president of the ISBA Advisory Board, following up from Professor Elizabeth (Liz) Wellington (Warwick), who is thanked for her great contribution to the community. Once the new Advisory Board is in full operation it will be time to select the new venue, which will take place in Europe in the summer of 2014. We hope that it will be as invigorating and fun as this meeting has been. 